\title{
Revisión al Desarrollo de la Tecnología de Radio Cognitiva en Bandas VHF - UHF en el Ecuador
}

\section{Review of the Development of VHF - UHF Bands Cognitive Radio Technology in Ecuador}

\author{
Andy Vega \\ Universidad Nacional de Loja \\ Loja, Ecuador \\ andy.vega@unl.edu.ec \\ Orcid: 0000-0003-0106-6880
}

\begin{abstract}
Resumen- La paulatina demanda, sobreutilización y subutilización espectral son factores que han denotado, a nivel gubernamental y de investigación, una búsqueda de soluciones legales y tecnológicas para optimizar el uso del espectro radioeléctrico. Bajo este argumento, el documento aborda una revisión al estado del arte sobre la aplicación de la tecnología de Radio Cognitiva (RC) sobre redes de sensores inalámbricos cognitivos (CWSN, Cognitive Wireless Sensor Networks), o de reúso espectral para la explotación de forma mejorada del espectro radioeléctrico en bandas VHF - UHF en el Ecuador. Esta recopilación u observación permitiría identificar el estado del arte para el desarrollo del proyecto de investigación "Smart UNL", que pretende desplegar una red de sensores inalámbricos basados en Radio Cognitiva (RC), para control de luminarias dentro de un Smart Campus. Los resultados evidencian cero aprovechamientos del concepto fundamental de RC para este tipo de aplicaciones, ya que los trabajos investigados se orientan a otro tipo de estudios de telecomunicaciones RC, lo que entrevé un amplio campo de aplicación y desarrollo de CWSN para una posible gestión y control de componentes de un Smart Campus.
\end{abstract}

Palabras Clave: Telecomunicaciones, Radio, Cognitiva, Frecuencia, Espectro, Inalámbricas.

\begin{abstract}
Review of the Development of VHF-UHF Band Cognitive Radio Technology in Ecuador Gradual demand, overuse and under-utilization of the spectrum are factors that have denoted, at the government and research levels, a search for legal and technological solutions to optimize the use of the radio spectrum. Under this argument, the document addresses a review of the state of the art on the application of Cognitive Radio (CR) technology on Cognitive Wireless Sensor Networks (CWSN) or spectral reuse for the improved exploitation of the radio spectrum in VHF - UHF bands in Ecuador. This collection or observation would allow the identification of the state of the art for the development of the research project "Smart UNL" that intends to deploy a network of wireless sensors based on Cognitive Radio (CR) for control of luminaires within a Smart Campus. The results show that the fundamental concept of CR for this type of application is not being exploited since the researched work is oriented towards other types of CR telecommunications studies, which suggests a wide field of application and development of CWSN for the possible management and control of components of a Smart Campus.
\end{abstract}

Sumario: I. Introducción, II. Radio cognitiva (RC), III. Radio cognitiva en el mundo, IV. Redes de sensores inalámbricos, V. Contexto de radio cognitiva en Ecuador para Smart Campus. Una revisión, VI. Conclusión y trabajo futuro.

Como citar: Vega, Andy. (2020). Revisión al Desarrollo de la Tecnología de Radio Cognitiva en Bandas VHF UHF en el Ecuador. Revista Tecnológica - Espol, 32(1). Recuperado a partir de http://www.rte.espol.edu.ec/index.php/tecnologica/article/view/680 


\section{INTRODUCCIÓN}

El desarrollo de las telecomunicaciones a nivel mundial representa una reingeniería continua de sus aspectos técnicos, administrativos y legales. La gestión del espectro radioeléctrico no deja de ser un tema de gran interés para los gobiernos de turno y, en particular, para los entes técnicos responsables de su administración y control. Será entonces de gran importancia desarrollar mecanismos tecnológicos que puedan ser partícipes de una mejora en la explotación espectral, aprovechando las instancias de subutilización o infrautilización de frecuencias que, medios como la radio y televisión, ocasionan durante su normal operación. Los sistemas de Radio Cognitiva (RC) sin duda se han establecido como una tentativa de aplicación en las tecnologías de telecomunicaciones; por ser una forma de comunicación inalámbrica, en la que un transceptor inteligente puede detectar los canales de comunicación que se están utilizando y los que no se están utilizando, y al instante pasar a los canales no empleados, evitando al mismo tiempo los ocupados. Esto optimiza el uso del espectro de radiofrecuencias (RF) y la posibilidad de reducir al mínimo la interferencia a otros usuarios [2]. En la actualidad, el desarrollo de los sistemas cognitivos ha tenido un moderado despliegue, derivado de condiciones de carácter mundial donde las presiones de los diferentes mercados para establecer estándares y normalizaciones se hace cada vez más fuerte y, donde a su vez, se debe mantener flexibilidad suficiente para permitir la innovación tecnológica de empresas, compañías e instituciones. Ante este escenario, la Universidad Nacional de Loja a través del proyecto "Smart UNL", busca posicionar su propia arquitectura Smart donde además se investiga la posibilidad de implementar técnicas de comunicaciones apegadas a la optimización espectral como lo considera RC, todo esto originado por la penetración de las redes inalámbricas en nuestra sociedad, habiendo previsiones de crecimiento muy elevadas con la irrupción del Internet de las Cosas (IoT). Sin embargo, esta interconexión masiva desboca en la aparición de inconvenientes como son primordialmente la saturación del espectro radioeléctrico o las interferencias provocadas entre sistemas, lo que repercute en la calidad del servicio y por lo tanto supone un problema para la conexión de elementos. Será entonces importante recapitular el contexto de los sistemas de Radio Cognitiva, conocer su penetración, aplicación y desarrollo actual, principalmente en el Ecuador, con el afán de aplicarla dentro del diseño y despliegue de redes de sensores inalámbricos, que, dicho sea de paso, al lograr su funcionamiento pueden ser útiles para la gestión y control de un sin número de componentes IoT en los denominados Smart Campus.

\section{RADIO COGNITIVA (RC)}

La necesidad de innovación en diferentes sistemas de telecomunicaciones, demandadas por las nuevas necesidades sociales, provocó la intromisión de novedosos conceptos y tecnologías para soportar el avance de las comunicaciones, y específicamente, en los sistemas inalámbricos. Así, Joseph Mitola III en 1991 involucró el término Radio definido por Software (SDR o Radio Software), con el objeto de referirse a un tipo de radios configurables y reprogramables, equipos donde elementos de hardware son capaces de realizar diferentes funciones en distintos instantes de tiempo, con la introducción de cambios en su configuración mediante software [2]. El concepto SDR surge para solucionar parcialmente inconvenientes de compatibilidad e interoperabilidad en redes inalámbricas, ya que establecen un conjunto de procedimientos y técnicas orientadas a realizar el procesamiento de señales de información por medio de un dispositivo de propósito general, el cual puede ser modificado mediante software, logrando así un cambio dinámico, automático y eficiente entre tecnologías, sin tener que incurrir en gastos/costes por concepto de nuevos equipos y evitando incrementos considerables en el tamaño de éstos [4].

\section{1.- Redes de Radio Cognitiva - (CRNs)}

Las CRNs, o redes de acceso dinámico al espectro (Dynamic Spectrum Access Networks, DSANs), brindan un gran ancho de banda a los usuarios móviles a través de arquitecturas heterogéneas inalámbricas y técnicas de acceso dinámico al espectro (Ver Figura 1). El uso eficiente del espectro existente puede mejorarse, a través del acceso oportunista por usuarios secundarios, a bandas con licencia o sin licencia, donde se asegura la no creación de interferencias. Sin embargo, las CRNs imponen retos a la investigación debido a los problemas técnicos que aún se presentan, donde varios de estos inconvenientes requieren de soluciones eficientes.

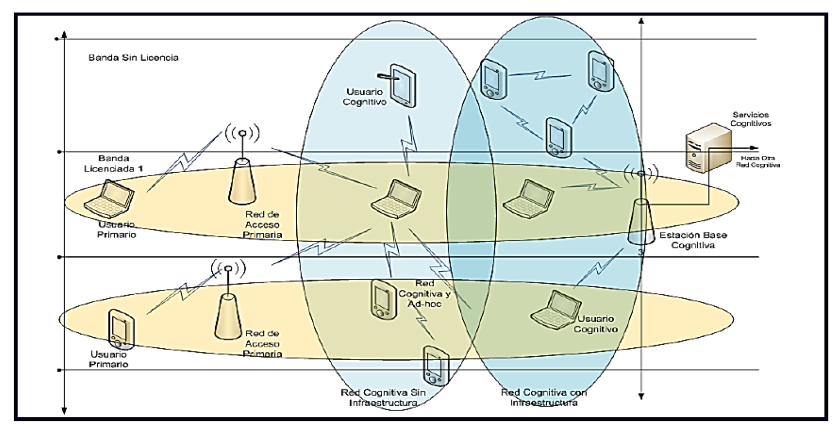

Figura 1.- Arquitectura de una CR

\section{2.- Capacidad Cognitiva}

La capacidad cognitiva de una RC permite la interacción, en tiempo real, con un ambiente de radiofrecuencia para determinar parámetros de comunicación apropiados y, adaptarse al entorno radio de modo dinámico. Los pasos requeridos se muestran en la figura 2 y se los conoce como ciclo cognitivo.

* Detección del Espectro. - La RC analiza las bandas de espectro disponibles, recoge su información, y después detecta los white spaces (espacios vacíos o huecos espectrales) en el espectro.

* Análisis del Espectro. - Consiste en evaluar las características de los huecos espectrales que han sido detectados en el primer paso.

* Decisión Espectral. - La RC determina la tasa de datos, el modo de transmisión y el ancho de banda de transmisión. 
* Después la banda de espectro apropiada se escoge teniendo en cuenta las características del espectro y los requerimientos de los usuarios [5].

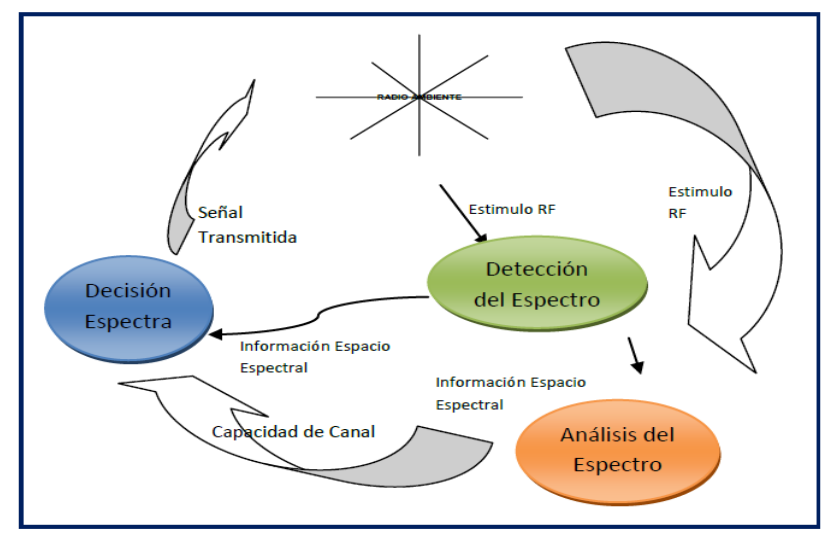

Figura 2.- Ciclo cognitivo

\section{3.- Detección de Espectro}

Los sistemas de Radio Cognitiva se muestran como una alternativa tecnológica para el uso eficiente del espectro radioeléctrico. $\mathrm{Su}$ capacidad de detectar partes del espectro, que están o no siendo usadas por las diferentes señales emitidas por usuarios múltiples y legítimos de los servicios de telecomunicaciones, la hacen capaz de transmitir en bandas de frecuencia como televisión y radiodifusión. Para ello existe una clasificación de usuarios: los primarios y los secundarios. Los usuarios secundarios pueden tener acceso y compartir el espectro si los usuarios primarios están inactivos o, en caso de que los niveles de señal primaria emitida sean insignificantes para la recepción [2]. Existen tres técnicas de detección del espectro: detección de energía, detección por filtro adaptado y detección ciclo estacionaria. Cada una de ellas ofrece menor o mayor complejidad de operación y así mismo baja o alta capacidad de detección. El uso de cada una de ellas depende de la cantidad de información disponible, referente a las señales primarias que se encuentran en el aire.

\section{RADIO COGNITIVA EN EL MUNDO}

Con motivo de buscar eficiencia en el uso del espectro, alrededor del planeta se han desarrollado varias propuestas de arquitectura radio cognitiva. Por ejemplo, CR1 desarrollada por Joseph Mitola, se basa en la utilización de un lenguaje de representación del conocimiento de radio para establecer las adaptaciones de la radio RKRL (Radio Knowledge Representation Language). Este lenguaje simboliza la noción de radio de un conjunto de lenguajes naturales y modelos basados en computador [6].

Así mismo, $\mathrm{xG}$ es un concepto de Sistema y Tecnología desarrollada por el DoD (Departamento de Defensa de los Estados Unidos) para el acceso dinámico a todos los recursos de frecuencia. Las redes Ad-Hoc y su crecimiento despiadado, con topologías dinámicas y cada vez más complejas, estimuló la necesidad de instituir nuevas alternativas de acceso al espectro. Por lo tanto, la tecnología XG se muestra conveniente para la implementación de arquitecturas de red centralizadas o no, mostrando flexibilidad con respecto al límite de interferencia deseado. Es importante resaltar el estándar IEEE 802.22, como el primero basado en tecnología de radio cognitiva, conocido como WRAN (Wireless Regional Área Networks). El objetivo preponderante es el acceso al ancho de banda en áreas rurales o remotas con un alto rendimiento, incluso comparable con tecnologías de acceso a banda ancha existentes en la actualidad [8] y [9].

Finalmente, distintos sistemas basados en redes de radio cognitiva han aparecido como alternativas de mejora en el aprovechamiento del espectro y su explotación. Adapt4, CORTEKS, Spectrum pooling, Corvus, Kuar, SPARTA, KNOWS, OCRA, entre otros, son parte de un grupo de tecnologías propietarias fundamentadas en la CR. Su desarrollo aún es limitado.

\section{REDES DE SENSORES INALÁMBRICOS COGNITIVOS}

Las redes de sensores inalámbricos cognitivos tienen como objeto funcionar dentro de espacios frecuenciales que mantienen o no usuarios primarios activos, y de esta forma apoyan a la compartición de espectro de usuarios inalámbricos, con licencia y sin licencia, dentro de una misma área geográfica. Como es de esperarse, los mayores retos radican en la no interferencia de los usuarios primarios que usan su red, y además en brindar una fina distribución de tiempo y frecuencia a los usuarios secundarios.

Existen algunos proyectos que se han dedicado a manejar este concepto innovador y original. SENDORA, por ejemplo, consiste en desarrollar un "Sensor de Redes apoyado en una Radio Cognitiva", que ayudará a resolver este problema gracias a la introducción de redes de sensores y capacidades de redes asociadas. El proyecto WISERNETS (Radio cognitiva de Software) tiene como objetivo principal describir los límites fundamentales y el diseño de nuevos algoritmos, para la detección del espectro distribuido en WSN radio asistida cognitiva, lo que le permite tomar decisiones dependiendo de dónde están los claros espectrales o viceversa.

Todos estos proyectos, y algunos otros basados en RC, pero no pensados para el control de dispositivos en un Smart Campus, mantienen un punto de vista técnico, donde se proponen técnicas innovadoras en detección de espectros y de actuación para identificar claros espectrales, considerando la coexistencia de radios cognitivas con tecnología primaria autorizada, sin forjar interferencias dañinas. Finalmente, el diseño, la dimensión y la conexión de las redes inalámbricas de sensores están tratados a fondo.

\section{CONTEXTO DE RADIO COGNITIVA EN ECUADOR PARA SMART CAMPUS. UNA REVISIÓN}

La aceptación e incorporación de nuevas y variadas tecnologías de telecomunicaciones en nuestro país se han desarrollado de manera progresiva y perseverante, y las redes de fibra óptica y telefonía celular como los principales nichos de proyección de las comunicaciones del país. Otras aplicaciones tecnológicas que ofrecen servicios de telecomunicaciones son los sistemas inalámbricos de banda ancha WI-FI y CDMA 450, que por 
ejemplo son usadas para servir múltiples usuarios con voz y datos dando mayor énfasis en sitios remotos o rurales.

Por otra parte, las tecnologías para enlaces inalámbricos basadas en Radio Cognitiva en fase de explotación no aparecen, encontrándose únicamente en etapas de estudio o investigación. Con la intención de ejecutar una búsqueda minuciosa se consulta los repositorios bibliotecarios de los principales centros de educación e investigación superior del Ecuador, los mismos que se encuentran en la capacidad de aportar con investigaciones que vinculen a la tecnología RC. Como se mencionó anteriormente, la intención de este trabajo será evidenciar si el concepto de Radio Cognitiva ha logrado ser usado en Redes de Sensores Inalámbricos Cognitivos, con el afán de su aplicación en el control y gestión de infinidad de componentes en un Smart Campus.

Un primer análisis de los Sistemas de Radio Cognitiva en el Ecuador se desarrolló en el año 2006 bajo el proyecto de titulación denominado "Análisis del Estándar IEEE 802.22 (Wireless Regional Área Network (WRAN)) y su posible implementación en el Ecuador"; ahí se expresan los conceptos del sistema inicial WRAN y las exigencias funcionales como la base para la creación de ese estándar. A lo largo del estudio se muestran los requerimientos obligatorios que hacen posible que un sistema WRAN pueda actuar en un ambiente proveedor de servicios de Internet. Así mismo, se da un ejemplo de análisis sobre la implementación de una estación base WRAN en áreas rurales y remotas de la provincia de Loja, donde el acceso de tecnologías de banda ancha por cable es completamente limitado, y es donde se concluye que la elección de una tecnología inalámbrica es lo más factible [10].

Más adelante, en el año 2010 en la Escuela Politécnica Nacional (EPN) se presenta un nuevo trabajo vinculado a los sistemas de Radio Cognitiva, denominado "Análisis de Factibilidad Para la Utilización de Cognitive Radio (Radio Cognitiva) en las Radiocomunicaciones necesarias para casos de Emergencia en el Ecuador"; luego de una revisión general de los sistemas de Radio Cognitiva, el autor efectúa un estudio de los principales aspectos técnicos de SDR (Radio definido por Software) que reconocen la reconfigurabilidad de los parámetros de operación de Radio Cognitiva, para consecutivamente constituir las capacidades y ventajas técnicas que involucran su utilización en las radiocomunicaciones utilizadas en emergencias. Dicho estudio se complementa con el diseño de un sistema de radiocomunicaciones basado en RC y su distribución para servir en la ciudad de Quito, de forma que incorpora de manera práctica el alcance y ventajas que ofrece esta tecnología [11].

Posteriormente, se han ejecutado dos trabajos adicionales establecidos como factibilidades técnicas de implementación. El primero en el año 2014, denominado "Análisis de factibilidad técnica en la implementación de una red WRAN (IEEE 802.22) en escuelas y colegios fiscales de sectores rurales y urbanos marginales de la Provincia del Guayas", donde se detalla el diseño de una Red WRAN (Wireless Regional Área Network), establecida bajo el estándar IEEE 802.22 y que, de acuerdo con los resultados obtenidos, en caso de llevarse a cabo, permitiría proporcionar a escuelas y colegios fiscales de sectores marginales de la Provincia del Guayas servicios de telecomunicaciones [13]; y el segundo que explica una solución tecnológica basada en WRAN - 802.22 para la automatización de un sistema de lectura remota de los medidores de energía eléctrica, denominado "Factibilidad técnica de implementación de Smart Metering en zonas rurales con Tecnologías de Radio Cognitiva en los espacios en blanco de televisión" [14].

Otro estudio de relevancia se presenta dentro de la región norte de nuestro país y se encuentra expuesto en el documento "Sensing TVWS with open source technology in Ecuador", el cual construye la caracterización de los espacios en blanco de la televisión (TVWS) como parámetro principal para concientizar sobre la existencia de espectro radio eléctrico subutilizado, el cual podría ser utilizado para solventar a través de reúso espectral las necesidades de conectividad de zonas desfavorecidas [15].

Para el año 2015, en la Escuela Superior Politécnica del Litoral (ESPOL) se presenta el trabajo "Sensado de Espectro de Banda Ancha Basado en Muestreo SubNyquist para Radio Cognitivo"; el trabajo únicamente tiene como objetivo realizar la identificación de las posibles oportunidades espectrales, basado en la implementación de la función de sensado de espectro de banda ancha, utilizando muestreo sub_nyquist para sistemas de radio cognitivo. En esta misma institución también se trabaja en el proyecto: "Diseño de un Algoritmo de predicción de canales disponibles en el rango de frecuencia asignada a televisión basado en Markov, para lograr acceso dinámico al espectro en ambientes indoor en zonas Urbanas", que se enfoca en el diseño de un algoritmo para $\mathrm{n}$ canales conjuntos en la banda de televisión, que permite el análisis predictivo de disponibilidad en canales conjuntos, con la finalidad de asistir en el uso óptimo de sistemas de acceso oportunistas al espectro, los cuales son de gran utilidad para solventar la problemática que se ha presentado en los últimos años en cuanto a la saturación del espectro radioeléctrico.

En el artículo "Análisis del método matemático de detección SVD en cognitive radio" se estudia el desempeño del método de descomposición en valores singulares (SVD), aplicado a la detección de usuarios en una red móvil cognitiva.

En este otro artículo "Estado del Arte, Radio Cognitiva para Operador de Red Virtual en la Infraestructura de Medición Avanzada", realizado por Marcelo Camacho Vera y Esteban Inga Ortega, también involucra a RC en su investigación; este trabajo se fundamenta en el uso de tecnologías de telecomunicaciones RC como un aporte para fortalecer y mejorar los sistemas eléctricos de medición avanzada (AMI) o Smart Grid, que se encarga de la medición inteligente de energía eléctrica. El objetivo es valerse de un operador móvil de red virtual para reducir los costos por arrendamiento del espectro de frecuencia primario [16].

Nuevamente en la EPN, se realiza un nuevo estudio denominado: "Metodología para la localización de TV White Spaces en zonas rurales del Distrito Metropolitano de Quito mediante el uso del software de planificación de red ICS Designer"; este trabajo se efectúa en el año 2017 e implementa una metodología para representar la ubicación de los espacios en blanco de televisión (TV 
White Spaces) en áreas rurales del DMQ (Distrito Metropolitano de Quito), mediante el uso de una herramienta de planificación de radiofrecuencias. Para el 2018, se trabaja en una investigación que ejecuta una simulación de los algoritmos más utilizados (detección de energía, detección de características ciclo estacionarias de la señal y detección basada en la transformada Wavelet), con el fin de conocer cuál de ellos ofrece mejor desempeño en términos de precisión de detección y utilización de recursos; se titula: "Análisis y simulación de algoritmos para detección de espectro en radio cognitiva".

El artículo "Consideraciones para el despliegue de redes basadas en Radio Cognitiva: Ocupación y Regulación del Espectro", publicado en el mes de octubre del año 2019, presenta los fundamentos de RC, sus beneficios y desafíos, información sobre los estándares existentes de los sistemas de CR, así como algunos ejemplos de implementación de estos sistemas, que sugieren que el marco regulatorio y jurídico puede facilitar o dificultar las experiencias y avances en este campo [19].

Finalmente, aunque el listado de estudios en el Ecuador sobre la tecnología de Radio Cognitiva no es extenso, no se han expuesto la totalidad de las investigaciones o trabajos, ya que todos están referidos o coinciden en acciones de estudio similares: ocupación de espectro, simulaciones de sensado, acceso al medio inalámbrico, optimización de radiofrecuencias, diseño de algoritmos, etc.

\section{CONCLUSIÓN Y TRABAJO FUTURO}

Es evidente que los sistemas de Radio Cognitiva en nuestro país no han logrado tomar un protagonismo significativo, el mismo que sea aplicable en diferentes aspectos donde las telecomunicaciones basadas en los lineamientos de $\mathrm{RC}$ representen un aporte en muchos de los sectores sociales, productivos y académicos del Ecuador. Ya el ingeniero Danilo Corral, en el año 2011, propone la idea de crear e identificar una red piloto con 802.22, donde se sugiere hermanar instituciones participantes locales o externas; crear una estructura organizacional jerárquica; conseguir permisos por parte del ente regulador; efectuar pruebas en las diferentes regiones del país para observar el comportamiento del estándar 802.22; y, finalmente menciona construir un documento técnico que recoja los datos arrojados en las evaluaciones para la posterior discusión de los resultados [17]. Pese a las iniciativas, todo este vasto trabajo no ha llegado a consolidarse y, por tanto, el perfeccionamiento de las tecnologías de Radio Cognitiva en el Ecuador se mantiene como un tema abierto para su desarrollo.

Así mismo, se evidencia que, hasta el cierre de esta revisión de literatura, aún no se han generado temas de investigación o estudio que pretendan desarrollar redes de sensores inalámbricos cognitivos en el Ecuador. Pese a que en otros países ya se han creado algunos trabajos al respecto, queda latente la oportunidad pionera para contribuir con el despliegue de este tipo de sistemas y, mejor aún, consolidarlas dentro de aplicaciones IoT en un Smart Campus, como es la intención de la Universidad Nacional de Loja y su proyecto Smart UNL.

\section{REFERENCIAS}

[1] Arcotel. (2017). Artículo 7 de la Ley Orgánica de Transferencia y Acceso a la Información Pública - LOTAIP Literal a2) Base legal que la rige, Recuperado de http://www.arcotel.gob.ec/wpcontent/uploads/downloads/2017/02/a2-Base-legal-que-rige-a-lainstitucion-ENERO2017.pdf

[2] Manjarrez, I. (2010). Radio Cognitiva, (Tesis de Ingeniería), Universidad Nacional Autónoma de México. Recuperado de http://www.ptolomeo.unam.mx:8080/xmlui/bitstream/handle/132 $.248 .52 .100 / 894 / \mathrm{A} 5$. pdf? sequence $=5$

[3] Mitola, III. \& Maguire, Jr. (1999). Cognitive Radio: Making Software Radios More Personal. Personal Communications IEEE, 4(6), 13-18. doi: 10.110998.788210

[4] Ceballos, C. \& Betancour, L. SDR: La alternativa para la evolución inalámbrica a nivel fisico, Recuperado de: http://roboticslab.uc3m.es/publications/Articulo1.pdf

[5] Hinostroza, V. \& Barraza, A. (2012). Uso de Radio Cognitiva para Asignación Dinámica de Espectro en Bandas no licenciadas. (Tesis inédita de Maestría). Universidad Autónoma de ciudad Juarez.

[6] Mitola III, J. (2000) Cognitive Radio: An Integrated Agent Architecture for Software Defined Radio, Ph.D. Dissertation Royal Institute of Technology, Stockholm, Sweden archive/macros/latex/contrib/supported/IEEEtran/

[7] Rieser, C. (2004) Biologically Inspired Cognitive Radio Engine Model Utilizing Distributed Genetic Algorithms for Secureand Robust Wireless Communications and Networking, Ph.D. Dissertation, Virginia Tech "PDCA12-70 data sheet," Opto Speed SA, Mezzovico, Switzerland.

[8] Cordeiro, C. Challapali, K. Birru, D. and Shankar, S. (2005) IEEE 802.22: the first worldwide wireless standard based on cognitive radios, in Proc. IEEE

[9] IEEE 802.22. Working group on wireless regional area networks, Enabling Spectrum Sharing and Rural Broadband Wireless Access Using Cognitive Radio Technology in White Spaces Recipient of the IEEE SA

[10] Simancas, E. (2006) Análisis del Estándar IEEE 802.22 (Wireless Regional Area Network (WRAN)) y su posible implementación en el Ecuador, Disertación de Ingeniería, Escuela Politécnica Nacional.

[11] Segura, D. (2010) Análisis de Factibilidad Para la Utilización de Cognitive Radio (Radio Cognitiva) en las Radiocomunicaciones necesarias para casos de Emergencia en el Ecuador, Disertación de Ingeniería, Escuela Politécnica Nacional.

[12] Hidalgo, J. (2013) Análisis de Tecnologías Inalámbricas para mejorar el diseño de la red de Comunicaciones en el Sector Rural centro de Morona Santiago, Disertación de Maestría, Escuela Superior Politécnica del Chimborazo.

[13] Maggi, W. (2014) Análisis de factibilidad técnica en la implementación de una red WRAN (IEEE 802.22) en escuelas y colegios fiscales de sectores rurales y urbanos marginales de la Provincia del Guayas, Disertación de Maestría, Universidad Católica Santiago de Guayaquil.

[14] Guanoluisa, C . (2015) Factibilidad Técnica de Implementación de Samrt Metering en zonas rurales con Tecnologías de Radio Cognitiva en los espacios en blanco de televisión. Universidad Politécnica Salesiana Sede Quito.

[15] Matamoros, J., Corral, D., Pietrosemoli E. (2014) Sensing TVWS with open source technology in Ecuador. Universidad de las Fuerzas Armadas ESPE, Sangolquí - Ecuador; International Centre for Theoretical Physics, Trieste - Italy.

[16] Camacho, M \& Inga, E. (2015) Estado del Arte, Radio Cognitiva para Operador de Red Virtual en la Infraestructura de Medición Avanzada. Universidad Politécnica Salesiana Sede Quito.

[17] Corral, D. (2011) EL ESTÁNDAR 802.22. WALC 2011. Guayaquil - Ecuador.

[18] R. W. Brodersen (2004), "CORVUS: A Cognitive Radio Approach for Usage of Virtual Unlicensed Spectrum," White Paper, Berkeley Wireless Research Center.

[19] Carro, Gonzalo, Hernández, Patricia. Beltramelli, Federico, Simon, María, Capdehourat, Germán1, Rodríguez, Benigno. (2019). Consideraciones para el Despliegue de Redes basadas en Radio Cognitiva: Ocupación y Regulación del Espectro. Revista Politécnica, volumen 44, 21 - 31 